\title{
COMMISSION I8
}

Compte rendu de la séance. 3 septembre I955

Président: Prof. P. Tardi.

SECRÉTAIRE: Ingr A. Gougenheim.

\section{Résolution adoptée}

La Commission 18 a été créée en 1922 sous le nom de 'Commission des Longitudes par télégraphie sans fil' pour s'occuper plus spécialement des opérations de révision des longitudes mondiales. Elle a effectivement organisé et dirigé les opérations de 1926 et I933 et assuré la publication de leurs résultats.

La nouvelle opération de longitudes, qui doit avoir lieu en 1957-58 dans le cadre de l'Année Géophysique Internationale, offrira un caractère très différent de celui des opérations précédentes; elle réalisera essentiellement une détermination d'ensemble, de très haute précision, des longitudes des observations constituant le 'Système du Bureau International de l'Heure'; elle doit par suite être rattachée plus particulièrement à la Commission 3I (Commission de l'heure) et au Comité spécial pour l'Année Géophysique Internationale (C.S.A.G.I.).

Dans ces conditions, la Commission 18 considère comme terminée la partie essentielle de la tâche pour laquelle elle avait été créée et qu'elle peut, sans inconvénient, être dissoute. Elle reconnaît, en ce qui concerne les déterminations ordinaires de positions géographiques, que ces questions sont surtout du ressort de la Section d'Astronomie géodésique de l'Association internationale de Géodesie. Elle estime que l'Union Astronomique Internationale doit entretenir une liaison étroite avec cette Section d'Astronomie géodésique, sans qu'il soit cependant nécessaire que l'U.A.I. possède en son sein un organisme équivalent.

La Commission I8 demande, à l'unanimité que ceux de ses membres qui n'appartiennent à aucune autre Commission de 1'U.A.I. soient répartis entre les Commissions 19 et 3I, sous réserve d'un accord préalable à obtenir des organismes nationaux interessés. 